\title{
Targeting the Warburg effect to overcome lapatinib resistance in esophageal adenocarcinoma
}

\author{
Samantha Holmes ${ }^{1}$, Md Sazzad Hassan ${ }^{1,2}$, Victoria Makuru¹, Urs von Holzen ${ }^{1,2,3}$ \\ ${ }^{1}$ Indiana University School of Medicine-South Bend, South Bend, IN 46617, \\ ${ }^{2}$ Harper Cancer Research Institute, South Bend, IN 46617, ${ }^{3}$ Goshen Center for \\ Cancer Care, Goshen, IN 46526
}

Background: Esophageal adenocarcinoma (EAC) overexpresses HER2. Lapatinib, a dual tyrosine kinase inhibitor blocking HER1 and HER2 pathways failed to improve patient survival. Molecular mechanisms of this lapatinib resistance are still unclear. We therefore explored the role of glycolytic enzyme pyruvate kinase M2 (PKM2), a key regulator of the Warburg effect in the lapatinib resistance mechanism of EAC cells. Methods: First we established a lapatinibresistant OE19 (LPR-OE19) cell line from OE19 EAC cells. We then investigated the comparative cell growth inhibition, apoptotic and lactate production effects of HER2 inhibitor lapatinib and PKM2 inhibitor shikonin either alone or in combinations, both in OE19 and LPR-OE19 cells with and without knockdown of PKM2 and HSP40 by siRNAs. Results: Lapatinib resistant LPR-OE19 cells showed downregulation of HSP40 both at protein and mRNA levels, but showed upregulation of PKM2 only at the protein level. LPR-OE19 cells showed significantly reduced sensitivity to lapatinib induced cell growth inhibition, apoptosis and decreased cell migration compared to parent OE19 cells. Interestingly, augmented cell growth inhibition, apoptosis and decreased cell migration were observed in LPR-OE19 cells compared to parent OE19 cells when lapatinib was combined with shikonin. Knockdown of PKM2 in LPR-OE19 cells abolished the reduced sensitivity of lapatinib induced cell growth inhibition, and also abolished augmented cell growth inhibition response when lapatinib and shikonin were combined. Contrary to that, knockdown of HSP40 in OE19 cells enhanced PKM2 expression leading to significantly reduced sensitivity to lapatinib induced growth inhibition with augmented growth inhibition when lapatinib and shikonin were combined. Moreover, LPR-OE19 cells showed enhanced lactate production compared to parent OE19. Interestingly, PKM2 and HSP40 co-localize with each other in the cell nucleus suggesting that PKM2 binds to molecular chaperone HSP40. Conclusions: These data suggest that targeting HSP40PKM2 interaction could be an innovative therapeutic approach to overcome lapatinib resistance in EAC. 